\title{
ASO Author Reflections: The Special Theory as an Entrance to the General Theory of Hepatoma Surgery
}

\author{
Junichi Shindoh, MD, $\mathbf{P h D}^{1,2}$ \\ ${ }^{1}$ Hepatobiliary-Pancreatic Surgery Division, Department of Gastroenterological Surgery, Toranomon Hospital, Minato-ku, \\ Tokyo, Japan; ${ }^{2}$ Okinaka Memorial Institute for Medical Disease, Tokyo, Japan
}

\section{PAST}

Anatomic resection (AR) of the tumor-bearing portal territories was originally proposed as a theoretically optimal surgical maneuver for hepatocellular carcinoma (HCC $)^{1}$ in which microscopic cancer spread via the closest portal branches is frequently observed. While a number of studies have been reported so far, the true efficacy of AR remains under debate because of a heterogeneity in study populations, lack of standardization of surgical maneuvers, and difference in quality control among past studies. The University of Tokyo group previously attempted to clarify the clinical impact of AR, focusing on a highly selected population with primary, solitary $\mathrm{HCC}$, measuring up to $5 \mathrm{~cm}$ in diameter, which was confined to one Couinaud's segment and treatable either by AR or non-AR. With strict quality control of surgical maneuver and statistical analysis, it was shown that AR is advantageous in reducing the incidence of local/unresectable recurrence, resulting in prolonged cancer-specific survival. ${ }^{2}$

\section{PRESENT}

In the present study, our group validated the aforementioned outcomes using the same methodology in selection of the study population and propensity score adjustment. ${ }^{3}$ Also, we have further clarified the differences in time to interventional failure and transition rate from the early

(C) Society of Surgical Oncology 2020

First Received: 7 July 2020

Accepted: 7 July 2020;

Published Online: 23 July 2020

J. Shindoh, MD, PhD

e-mail: shindou-tky@umin.ac.jp recurrence stage to advanced stages according to the choice of surgical maneuver, using a Markov model. The present analysis indicated that initial choice of surgical maneuver has significant influence on the pattern of recurrence and post-progression clinical course that may affect overall survival of patients with HCC.

\section{FUTURE}

Although the present study clearly indicated that AR is oncologically advantageous in reducing recurrence and delaying long-term stage progression after resection of $\mathrm{HCC}$, this is just a special theory in a specific population. It remains inconclusive whether or not the same scenario can be applied for recurrent lesions, multiple HCCs, or larger HCCs occupying 2 or more Couinaud's segments. Further studies are needed to optimize surgical approach for HCC based on the various clinical situations.

FUNDING None.

DISCLOSURES The authors declare that they have no conflict of interest.

\section{REFERENCES}

1. Makuuchi M, Hasegawa H, Yamazaki S. Ultrasonically guided subsegmentectomy. Surg Gynecol Obstet. 1985; 161: 346-50.

2. Shindoh J, Makuuchi M, Matsuyama Y et al. Complete removal of the tumor-bearing portal territory decreases local tumor recurrence and improves disease-specific survival of patients with hepatocellular carcinoma. J Hepatol. 2016; 64: 594-600.

3. Shindoh J, Kobayashi Y, Umino R et al. Successful anatomic resection of tumor-bearing portal territory delays long-term stage progression of hepatocellular carcinoma. Ann Surg Oncol. 2020. h ttps://doi.org/10.1245/s10434-020-08927-3.

Publisher's Note Springer Nature remains neutral with regard to jurisdictional claims in published maps and institutional affiliations. 